\title{
Influence of Zinc Oxide Nanoparticles on Cadmium Toxicity on Germination of Faba Bean (Vicia faba L.)
}

\author{
Salah M. H. Gowayed ${ }^{* 1,2}$ and Naif M. Kadasa ${ }^{2}$ \\ ${ }^{1}$ Botany Department, Faculty of Agriculture, Suez Canal University, Ismailia, Egypt \\ ${ }^{2}$ Biology Department, Faculty of Science, University of Jeddah, KSA
}

Received: $22 / 11 / 2015$

\begin{abstract}
This work aimed to study the influence of zinc oxide nanoparticles (ZnONPs) on Cadmium (Cd) toxicity on germination of Faba bean (Vicia faba L.). The results indicated that, the levels of $\mathrm{Zn}$ and Cd significantly increased in shoot and root in a correlation with their dose. The levels of shoot and root $\mathrm{Cd}$ decreased in plants treated with the increasing of ZnONPs dose. ZnONPs and/ or Cd increased the shoot length, shoot fresh weight and shoot dry weight and whereas decreased root length, root length /shoot length and root dry weight in comparison with the control. ZnONPs and/ or Cd decreased the Mean Daily Germination MDG, Germination Index GI, Coefficient of Velocity of Germination CVG, Promptness index PI, Germination stress tolerance index GSI, Seedling vigor index SVI and Root length stress tolerance index RLSI whereas increased Mean Germination Time MGT in comparison with control. ZnONPs at $250 \mathrm{mg} \mathrm{L}^{-1}$ with Cd $50 \mathrm{mg} \mathrm{L}^{-1}$ showed the highest decrease in MDG, GI, CVG, PI and GSI whereas MGT recorded highest values. The highest decreased in SVI was shown in ZnONPs at $500 \mathrm{mg} \mathrm{L}^{-1}$ with Cd $25 \mathrm{mg} \mathrm{L}^{-1}$. Meanwhile, the highest decreased in RLSI was shown in ZnONPs at $1000 \mathrm{mg} \mathrm{L}^{-1}$ with Cd $50 \mathrm{mg} \mathrm{L}^{-1}$. It can be concluded that, ZnONPs decreased Cd level in the seedling of Faba bean, as well as germination parameters whereas increased some growth parameters.
\end{abstract}

Keywords: ZnONPs, Cd toxicity, Faba bean, Germination.

\section{INTRODUCTION}

Faba bean (Vicia faba L.) is one of the most important seed crops in the world. It is an important protein-rich food that provides a wide number of populations in the developing countries as a source of cheap protein, thus partly recovering the large deficiency in animal protein sources (Safaa and Leur 2011).

Zinc $(\mathrm{Zn})$ is representative the second most plentiful transition metal in organisms after iron (Auld 2001). $\mathrm{Zn}$ is a fundamental micronutrient for plants, animals and humans. Generally, $\mathrm{Zn}$ absorbed as cation $\left(\mathrm{Zn}^{2+}\right)$ in plants, which works as the metal component or as a functional structure or a regulatory co-factor of many enzymes (Prasad et al., 2012).

$\mathrm{Cd}$ is a highly toxic heavy metal which induces oxidative stress in plants (Hasan et al., 2009). It is mainly entered to the ecosystem through mining and smelting of $\mathrm{Zn}$, industry and the application of phosphate fertilizers (Waisberg et al., 2003). Cd ions are readily taken up by the roots (Fargašová, 2004) and accumulated in different parts of the plant which cause various phytotoxic symptoms such as inhibition in root, shoot growth (Eshghi et al., 2010) by inhibiting cell division and the growth of cells or both of them (Pal et al., 2006). Moreover, it's change in morphological, physiological and biochemical characteristics in plants (Benavides et al., 2005; Talebi et al., 2014).

Although, $\mathrm{Zn}$ and $\mathrm{Cd}$ have different biological properties, there are many chemical and physical similarities. The association of $\mathrm{Cd}$ and $\mathrm{Zn}$ may be preventing the toxicity of $\mathrm{Cd}$ by $\mathrm{Zn}$. The convergence of $\mathrm{Cd}$ and $\mathrm{Zn}$ in environment may lead to several synergistic and antagonistic interactions in their uptake and tissue content (Jaouhra et al., 2011).

Nanotechnology is an evolutionary science and has introduced many novel applications in the many fields of science as biotechnology and agricultural industries.

*Corresponding author e-mail: salahgowed@yahoo.com
Nanoparticles (NPs) are molecular aggregates or atomic with size between 1 and $100 \mathrm{~nm}$ (Ball 2002; Roco 2003), that can sharply change their physical-chemical advantages compared to macro-molecules (Nel et al., 2006; Hediat, 2012). They have powerful advantages as a result of unique physical and chemical characteristics and huge surface area relative to the size, which give them the possibility to improve the life quality and contribute competitiveness in industry field (Homa and Aghili, 2014). However, as a result of their unique advantages, some researches have been done on the toxicological effect of NPs on plants, yet research focusing on the investigation of the beneficial effects of NPs on plants still incomplete. NPs can prospect to improve the nano-pesticide fertilizers, herbicides and genes, which target specific cellular organelles to release their content in plants (Siddiqui et al., 2015). Despite the much information available on the toxic effect of NPs in plant system, few studies have been conducted on mechanisms, by which NPs exert their effect on plant growth and development. In many studies, increasing evidence suggests that ZnONPs increase plant growth and development Siddiqui et al., 2015, peanut (Prasad et al., 2012), soybean (Sedghi et al., 2013), wheat (Ramesh et al., 2014) and onion (Raskar and Laware, 2014).

Seeds germination and seedling roots are sensitive stages in the plant growth circle and it is the critical stage of plants to the alteration in surrounding environment (Liu et al., 2011; Sfaxi-Bousbih et al., 2010). Thus, this stage is a best trend to study the toxicological mechanisms in plants by environmental contaminants (Sujing et al., 2012).

There are controversial reports about the effect of NPs on the growth and germination of plants (Mahajan et al., 2011). Therefore, the present work aimed to study the influence of ZnoNPs on Cd toxicity on germination parameters of Faba bean (Vicia faba L.).

Volume (4): 21-26 


\section{MATERIALS AND METHODS}

The present investigation was conducted under controlled condition in the Biology Lab, Biological Science Department, Faculty of Science, University of Jeddah, KAU during August 2015. Seeds of Faba bean (Vicia faba L.) cultivar Giza 2 were obtained from the Field Crops Research Institute, Agriculture Research Center, Giza, Egypt.

ZnONPs was obtained in the form of dispersion from Sigma-Aldrich, Steinheim, Germany (CAS Number 1314-13-2) of concentration 50 wt. \% in $\mathrm{H}_{2} \mathrm{O}$, average particle size (APS) was $<35 \mathrm{~nm}$. The particle size distribution (hydrodynamic diameter) was < $100 \mathrm{~nm}$ using dynamic light scattering (DLS) technique, $\mathrm{pH} 7 \pm 0.1$ (for aqueous systems) and density $1.7 \pm 0.1 \mathrm{~g}$ $\mathrm{mL}^{-1}$ at $25^{\circ} \mathrm{C}$.

Preparation of test solutions: Suspensions of ZnONPs in a concentration of 250,500 and $1000 \mathrm{mg} \mathrm{L}^{-1}$ were daily prepared with deionized water and dispersed with a sonicator (JL-360, Shanghai, USA) for $20 \mathrm{~min}$. 25 and $50 \mathrm{mg}$. $\mathrm{L}^{-1} \mathrm{Cd}$ solution as $3 \mathrm{CdSO}_{4} \cdot 8 \mathrm{H}_{2} \mathrm{O}$ were prepared with deionized water.

Seed preparation: 252 healthy and uniform size seeds of Faba bean were used in this study. The seeds were sterilizated using $2.5 \% \mathrm{NaOCl}$ solution according to Lin and Kao (1996), then washed three times with deionized water. 63 seeds (control and Cd groups) were immersed in water for 4 hours; the other seeds were divided in to three parts, and immersed in ZnONPs at concentrations 250, 500 and $1000 \mathrm{mgL}^{-1}$ for 4 hours.

Seed germination test: the seeds were placed in Petri dishes $90 \mathrm{~mm}$ (tri replicate) on filter paper then, add $5 \mathrm{ml}$ of ZnONPs suspensions (ZnONPs treated groups), $\mathrm{Cd}$ solution ( $\mathrm{Cd}$ treated groups) and deionized water for control group. All dishes were incubated in a growth room $\left(25 \pm 2^{\circ} \mathrm{C}, 65 \%\right.$ relative humidity and dark for $72 \mathrm{~h}$ then 14/10-h light/dark cycle) using complete randomize design. After that $7 \mathrm{ml}$ of treated solution add at second day, $10 \mathrm{ml}$ at fourth day and $10 \mathrm{ml}$ at sixth day for each Petri dish.

Germination parameters: Seeds becomes germinated when their radical extension exhibits longer than $3 \mathrm{~mm}$ (Prapatsorn et al., 2011). The germinated seeds were counted daily up to end the germination (4 days) for determination of Final Germination Percentage (FGP), Mean Germination Time (MGT), Germination Index (GI), Coefficient of Velocity of Germination (CVG) and Mean Daily Germination (MDG) was calculated according to Gowayed and Almaghrabi (2013).

Promptness index (PI), Germination stress tolerance index (GSI), Root length stress tolerance index (RLSI), Vigor index (SVI) calculated according to Raskar and Laware (2013) using following formulae:

- PI = nd2 (1.0) + nd4 (0.75) + nd6 (0.5) + nd8 (0.25)

Where $\mathrm{n}$ is the no. of seed germinated at day $\mathrm{d}$

- GSI $=$ PI of treated seeds $/$ PI of control seeds $\mathrm{x} 100$.

- SVI $=$ Germination $\% \times$ Seedling length $(\mathrm{cm})$.

- RLSI = Root length of treated seedling / Root length of control seedling $\mathrm{x} 100$.

Growth parameters: The growth parameters shoot length (SL), root length (RL), shoot fresh weight
(SFW), root fresh weight (RFW), shoot dry weight (SDW) and root dry weight (RDW) were measured at the end of the experiment after 8 days.

Analysis of heavy metals: At the end of exterminate shoots and roots of seedling were washed totally with distilled water and the samples oven dried at $78^{\circ} \mathrm{C}$ for three days. Dried samples were digested using a nitric $\left(\mathrm{HNO}_{3}\right)$-sulfuric $\left(\mathrm{H}_{2} \mathrm{SO}_{4}\right)$-perchloric $\left(\mathrm{HClO}_{4}\right)$ acid mixture (4:1:8 v/v) (Jackson 1973). The content of $\mathrm{Zn}$ and $\mathrm{Cd}$ was determined by the atomic absorption spectrophotometer (Thermo-electron, S Series GE 711838).

Statistical Analysis: Results were expressed as mean \pm SD (standard deviation). All data were subjected one way completely randomize ANOVA analysis to calculate the least significant difference (LSD) at $\mathrm{p}<0.05$ with Costat computer program.

\section{RESULTS AND DISCUSSION}

\section{a- Zinc and Cadmium determination:}

Germination and early seedling development assay has been regarded as a basic experiment for evaluating the toxic effect of any metal or chemical type of plants (Ahsan et al., 2007; Wang and Zhou, 2005). The toxicity of metal is an important evidence to evaluate germination and plant growth. Seedling growth is considered as an indicator of mental stress on the plant's ability to survive. Under heavy metal stress, the processes of germination, like embryo growth, will be depressed (Ahsan et al., 2007; Talebi et al., 2014). The chemical similarity for $\mathrm{Cd}$ and $\mathrm{Zn}$ in their association in ecosystem can lead to interaction between them (Jaouhra et al., 2011). In this study, concentrations of $\mathrm{Zn}$ and $\mathrm{Cd}$ were detected in Faba bean seedling (Table 1). $\mathrm{Zn}$ is detected in shoot and root of control plants, whereas $\mathrm{Cd}$ not detected. Also, we observed that the levels of heavy metals in the tested plants increased significantly with the increased concentration of ZnONPs application or Cd. It indicates that the ZnONPs are uptake and transported to the seedling due to increase in $\mathrm{Zn}$ content at conformable ZnONPs treatment. Moreover, increasing concentration of $\mathrm{ZnONPs}$ decrease concentration of $\mathrm{Cd}$ and the reverse is also observed. In general the content of the metal is multiplied in the root than shoot (Sresty and Rao, 1999; Homa and Aghili, 2014) in the same treatment. Hassen et al. (2005) found analogical results in them study in rice plant and reported that the increase level of $\mathrm{Zn}$ in culture medium leads to decrease in $\mathrm{Cd}$ absorption and the increasing content in roots with positive correlated in its content in shoot. The correlation between $\mathrm{Cd}-\mathrm{Zn}$ uptake may be is due to them competition (Hart et al., 2002; Jaouhra et al., 2011).

\section{b- Growth parameters:}

Data in (Table 2) indicated that ZnONPs and/ or Cd increased the shoot length, shoot fresh weight and shoot dry weight and decreased the root length, root length /shoot length and root dry weight in comparison with control.

For the transport in plant, shoot and root length are consider the important parameters (Sujing et al., 2012). The results in Table 2 showed that, the length of the 
shoots were increased while, the roots length were decreased in all treated plants. The increase of SL is lead to increase in SFW and SDW. In contrast, the inhibition of RL is lead to reduction in RFW, RDW and RL/SL.

Table (1): Zinc and cadmium concentrations in Vicia faba $\left(\mu \mathrm{g} . \mathrm{g}^{-1}\right)$ dry weight.

\begin{tabular}{|c|c|c|c|c|}
\hline \multirow{2}{*}{ Treatments (mg. $\mathrm{L}^{-1}$ ) } & \multicolumn{2}{|c|}{ Cd } & \multicolumn{2}{|c|}{$\mathbf{Z n}$} \\
\hline & Shoot & Root & Shoot & Root \\
\hline Control & $\mathrm{Nd}$ & $\mathrm{Nd}$ & $88.56 \pm 7.30^{\mathrm{h}}$ & $146.56 \pm 5.75^{\mathrm{h}}$ \\
\hline ZnONPs 250 & $\mathrm{Nd}$ & $\mathrm{Nd}$ & $377.56 \pm 1.95 \mathrm{f}$ & $3354.42 \pm 11.76^{\mathrm{f}}$ \\
\hline ZnONPs 500 & $\mathrm{Nd}$ & $\mathrm{Nd}$ & $573.25 \pm 6.53^{c}$ & $4917.60 \pm 571.52^{\mathrm{c}}$ \\
\hline ZnONPs 1000 & $\mathrm{Nd}$ & $\mathrm{Nd}$ & $1224.94 \pm 3.06^{\mathrm{a}}$ & $6687.57 \pm 1.73^{\mathrm{a}}$ \\
\hline Cd 25 & $10.24 \pm 0.66^{\mathrm{cd}}$ & $234.75 \pm 4.40^{\mathrm{e}}$ & $87.90 \pm 2.22^{h}$ & $145.89 \pm 1.50^{\mathrm{h}}$ \\
\hline Cd 50 & $18.70 \pm 2.40^{\mathrm{a}}$ & $671.20 \pm 5.74^{\mathrm{a}}$ & $89.34 \pm 3.74^{\mathrm{h}}$ & $146.96 \pm 2.11^{\mathrm{h}}$ \\
\hline ZnONPs $250+$ Cd 25 & $9.18 \pm 0.24^{\mathrm{d}}$ & $130.34 \pm 4.27^{\mathrm{f}}$ & $416.53 \pm 4.21^{\mathrm{e}}$ & $2816.14 \pm 5.52^{\mathrm{g}}$ \\
\hline ZnONPs $250+$ Cd 50 & $11.88 \pm 0.70^{b}$ & $401.83 \pm 1.57^{\mathrm{b}}$ & $247.19 \pm 57.16^{\mathrm{g}}$ & $2743.90 \pm 10.17^{\mathrm{g}}$ \\
\hline ZnONPs 500 + Cd 25 & $4.22 \pm 0.59^{\mathrm{e}}$ & $123.37 \pm 3.19^{f}$ & $353.21 \pm 1.91^{\mathrm{f}}$ & $3742.06 \pm 17.94^{\mathrm{de}}$ \\
\hline ZnONPs 500 + Cd 50 & $11.59 \pm 1.42^{\mathrm{bc}}$ & $329.22 \pm 0.95^{\mathrm{c}}$ & $272.69 \pm 0.69^{g}$ & $3503.68 \pm 6.95^{\mathrm{ef}}$ \\
\hline ZnONPs $1000+$ Cd 25 & $3.32 \pm 0.56^{\mathrm{e}}$ & $108.86 \pm 1.70^{\mathrm{g}}$ & $737.67 \pm 5.25^{\mathrm{b}}$ & $5236.94 \pm 13.88^{b}$ \\
\hline ZnONPs 1000 + Cd 50 & $9.92 \pm 0.88^{\mathrm{cd}}$ & $276.58 \pm 11.73^{d}$ & $456.21 \pm 5.05^{\mathrm{d}}$ & $3945.56 \pm 20.68^{d}$ \\
\hline
\end{tabular}

Data are means \pm SD of three independent experiments.

$\mathrm{a}, \mathrm{b}, \ldots$ or ${ }^{\mathrm{g}}$ indicated a significant difference at $\mathrm{p} \leq 0.05$.

Table (2): Effect of different concentration of ZnONPs and/or Cd on growth parameters.

\begin{tabular}{|c|c|c|c|c|c|c|c|}
\hline Treatments (mg. $\left.\mathrm{L}^{-1}\right)$ & RL & $\mathbf{S} \mathbf{L}$ & RL/SL & RFW & RDW & SFW & SDW \\
\hline Control & $\begin{array}{c}6.903 \pm \\
0.528^{\mathrm{a}}\end{array}$ & $\begin{array}{c}3.571 \pm \\
0.399^{\mathrm{b}}\end{array}$ & $\begin{array}{c}1.946 \pm \\
0.222^{\mathrm{a}}\end{array}$ & $\begin{array}{c}0.376 \pm \\
0.027^{\mathrm{a}}\end{array}$ & $\begin{array}{c}0.030 \pm \\
0.006^{\mathrm{a}}\end{array}$ & $\begin{array}{c}0.254 \pm \\
0.036^{\mathrm{b}}\end{array}$ & $\begin{array}{c}0.025 \pm \\
0.006^{\mathrm{c}}\end{array}$ \\
\hline ZnONPs 250 & $\begin{array}{c}3.440 \pm \\
0.277^{\mathrm{b}}\end{array}$ & $\begin{array}{c}5.060 \pm \\
0.752^{\mathrm{a}}\end{array}$ & $\begin{array}{c}0.688 \pm \\
0.106^{\mathrm{bc}}\end{array}$ & $\begin{array}{c}0.264^{ \pm} \\
0.024^{\mathrm{b}}\end{array}$ & $\begin{array}{l}0.027 \pm \\
0.006^{\mathrm{ab}}\end{array}$ & $\begin{array}{c}0.413 \pm \\
0.050^{\mathrm{a}}\end{array}$ & $\begin{array}{c}0.040 \pm \\
0.005^{\mathrm{a}}\end{array}$ \\
\hline ZnONPs 500 & $\begin{array}{l}2.916 \pm \\
0.367^{\text {bed }}\end{array}$ & $\begin{array}{l}4.471 \pm \\
0.632^{\mathrm{ab}}\end{array}$ & $\begin{array}{c}0.667 \pm \\
0.162^{\text {bc }}\end{array}$ & $\begin{array}{l}0.226 \pm \\
0.012^{\mathrm{bc}}\end{array}$ & $\begin{array}{c}0.020 \pm \\
0.002^{\mathrm{b}}\end{array}$ & $\begin{array}{l}0.355 \pm \\
0.059^{\mathrm{ab}}\end{array}$ & $\begin{array}{l}0.033 \pm \\
0.005^{\mathrm{abc}}\end{array}$ \\
\hline ZnONPs 1000 & $\begin{array}{c}2.796 \pm \\
0.177^{\mathrm{cd}}\end{array}$ & $\begin{array}{c}4.065 \pm \\
0.698^{\mathrm{ab}}\end{array}$ & $\begin{array}{c}0.706 \pm \\
0.163^{\text {bc }}\end{array}$ & $\begin{array}{c}0.178 \pm \\
0.008^{\mathrm{c}}\end{array}$ & $\begin{array}{l}0.022 \pm \\
0.007^{\mathrm{ab}}\end{array}$ & $\begin{array}{l}0.329 \pm \\
0.058^{\mathrm{ab}}\end{array}$ & $\begin{array}{c}0.036 \pm \\
0.007^{\mathrm{ab}}\end{array}$ \\
\hline Cd 25 & $\begin{array}{c}2.999 \pm \\
0.138^{\mathrm{bc}}\end{array}$ & $\begin{array}{c}3.675 \pm \\
0.430^{\mathrm{b}}\end{array}$ & $\begin{array}{c}0.824 \pm \\
0.102^{b}\end{array}$ & $\begin{array}{c}0.264 \pm \\
0.020^{\mathrm{b}}\end{array}$ & $\begin{array}{l}0.023 \pm \\
0.007^{\mathrm{ab}}\end{array}$ & $\begin{array}{l}0.328 \pm \\
0.042^{\mathrm{ab}}\end{array}$ & $\begin{array}{l}0.029 \pm \\
0.004^{\text {bc }}\end{array}$ \\
\hline Cd 50 & $\begin{array}{l}2.515 \pm \\
0.437^{\mathrm{cd}}\end{array}$ & $\begin{array}{l}4.037 \pm \\
0.523^{\mathrm{ab}}\end{array}$ & $\begin{array}{l}0.628 \pm \\
0.131^{b c}\end{array}$ & $\begin{array}{c}0.268 \pm \\
0.076^{\mathrm{b}}\end{array}$ & $\begin{array}{l}0.022 \pm \\
0.005^{\mathrm{ab}}\end{array}$ & $\begin{array}{l}0.332 \pm \\
0.029^{\mathrm{ab}}\end{array}$ & $\begin{array}{c}0.025 \pm \\
0.001^{\mathrm{c}}\end{array}$ \\
\hline ZnONPs $250+$ Cd 25 & $\begin{array}{l}3.070 \pm \\
0.302^{\mathrm{bc}}\end{array}$ & $\begin{array}{c}4.360 \pm \\
0.479^{\mathrm{ab}}\end{array}$ & $\begin{array}{c}0.708 \pm \\
0.078^{\mathrm{bc}}\end{array}$ & $\begin{array}{c}0.253 \pm \\
0.025^{\mathrm{b}}\end{array}$ & $\begin{array}{l}0.026 \pm \\
0.006^{\mathrm{ab}}\end{array}$ & $\begin{array}{l}0.348 \pm \\
0.054^{\mathrm{ab}}\end{array}$ & $\begin{array}{l}0.034 \pm \\
0.004^{\text {abc }}\end{array}$ \\
\hline ZnONPs $250+$ Cd 50 & $\begin{array}{l}2.620 \pm \\
0.079^{\mathrm{cd}}\end{array}$ & $\begin{array}{l}3.588 \pm \\
0.407^{\mathrm{ab}}\end{array}$ & $\begin{array}{c}0.736 \pm \\
0.072^{\mathrm{bc}}\end{array}$ & $\begin{array}{l}0.220 \pm \\
0.039^{\mathrm{bc}}\end{array}$ & $\begin{array}{l}0.023 \pm \\
0.004^{\mathrm{ab}}\end{array}$ & $\begin{array}{c}0.292 \pm \\
0.074^{\mathrm{b}}\end{array}$ & $\begin{array}{l}0.030 \pm \\
0.007^{\text {abc }}\end{array}$ \\
\hline ZnONPs $500+$ Cd 25 & $\begin{array}{l}2.798 \pm \\
0.454^{\mathrm{cd}}\end{array}$ & $\begin{array}{c}3.381 \pm \\
0.666^{b}\end{array}$ & $\begin{array}{l}0.858 \pm \\
0.246^{\text {bc }}\end{array}$ & $\begin{array}{c}0.257 \pm \\
0.018^{\mathrm{b}}\end{array}$ & $\begin{array}{l}0.026 \pm \\
0.007^{\mathrm{ab}}\end{array}$ & $\begin{array}{l}0.319 \pm \\
0.034^{\mathrm{ab}}\end{array}$ & $\begin{array}{l}0.034 \pm \\
0.007^{\mathrm{abc}}\end{array}$ \\
\hline ZnONPs 500 + Cd 50 & $\begin{array}{l}2.746 \pm \\
0.226^{\mathrm{cd}}\end{array}$ & $\begin{array}{c}4.398 \pm \\
0.579^{\mathrm{ab}}\end{array}$ & $\begin{array}{l}0.630 \pm \\
0.083^{b c}\end{array}$ & $\begin{array}{l}0.239 \pm \\
0.055^{\mathrm{bc}}\end{array}$ & $\begin{array}{l}0.021 \pm \\
0.005^{\mathrm{ab}}\end{array}$ & $\begin{array}{l}0.358 \pm \\
0.062^{\mathrm{ab}}\end{array}$ & $\begin{array}{l}0.037 \pm \\
0.004^{\mathrm{ab}}\end{array}$ \\
\hline ZnONPs $1000+$ Cd 25 & $\begin{array}{l}2.624 \pm \\
0.421^{\mathrm{cd}}\end{array}$ & $\begin{array}{l}4.332 \pm \\
1.510^{\mathrm{ab}}\end{array}$ & $\begin{array}{c}0.630 \pm \\
0.108^{\mathrm{bc}}\end{array}$ & $\begin{array}{c}0.173 \pm \\
0.067^{\mathrm{c}}\end{array}$ & $\begin{array}{l}0.027 \pm \\
0.001^{\mathrm{ab}}\end{array}$ & $\begin{array}{c}0.349 \pm \\
0.159^{\mathrm{ab}}\end{array}$ & $\begin{array}{l}0.037 \pm \\
0.011^{\mathrm{ab}}\end{array}$ \\
\hline ZnONPs $1000+$ Cd 50 & $\begin{array}{c}2.413 \pm \\
0.318^{\mathrm{d}}\end{array}$ & $\begin{array}{l}4.320 \pm \\
0.177^{\mathrm{ab}}\end{array}$ & $\begin{array}{c}0.557 \pm \\
0.052^{\mathrm{c}}\end{array}$ & $\begin{array}{l}0.214 \pm \\
0.038^{\mathrm{bc}}\end{array}$ & $\begin{array}{l}0.020 \pm \\
0.005^{\mathrm{ab}}\end{array}$ & $\begin{array}{l}0.305 \pm \\
0.029^{\mathrm{ab}}\end{array}$ & $\begin{array}{l}0.030 \pm \\
0.002^{\mathrm{bc}}\end{array}$ \\
\hline
\end{tabular}

Data are means \pm SD of three independent experiments.

$\mathrm{a}, \mathrm{b}, \ldots$ or ${ }^{\mathrm{g}}$ indicated a significant difference at $\mathrm{p} \leq 0.05$. 
These results in accordance with the results obtained by Pramod et al. (2011) who revealed that the higher dose of ZnONPs suspension reduced root and shoot growth of gram and mung seedlings, which may be duo to toxicity levels of NPs. ZnONPs significantly reduced the biomass of rye-grass, tip of root and different tissues of root. ZnONPs highly adhered on root surface and a few NPs were observed in the apoplast, steel and root endodermis. Zn transporter from root to shoot stay very low through ZnONPs treatments and the author indicated that the ZnONPs toxicity was not correlated directly with their bounded dissolution in the solution of bulk nutrient or rizosphere (Lin and Xing, 2008). So, this may explain the increasing of SL, SFW and SDW, although the inhibition of RL.

\section{c- Germination parameters:}

ZnONPs and/or Cd decreased the Mean Daily Germination MDG, Germination Index GI, Coefficient of Velocity of Germination CVG, Promptness index PI, Germination stress tolerance index GSI, Seedling vigor index SVI and Root length stress tolerance index RLSI and increased Mean Germination Time MGT in comparison with the control (Table 3).

Table (3): Effect of different concentration of ZnONPs and/or $\mathrm{Cd}$ on germination parameters.

\begin{tabular}{|c|c|c|c|c|c|c|c|c|c|}
\hline Treatments $\left(\mathrm{mg} . \mathrm{L}^{-1}\right)$ & FGP & MDG & CVG & MGT & GI & PI & GSI & SVI & RLSI \\
\hline Control & 100 & $\begin{array}{c}38.89 \pm \\
9.62^{\mathrm{a}}\end{array}$ & $\begin{array}{c}91.67 \pm \\
7.22^{\mathrm{a}}\end{array}$ & $\begin{array}{c}1.10 \pm \\
0.08^{\mathrm{c}}\end{array}$ & $\begin{array}{c}3.39 \pm \\
0.10^{\mathrm{a}}\end{array}$ & $\begin{array}{c}6.83 \pm \\
0.14^{\mathrm{a}}\end{array}$ & $\begin{array}{c}100.00 \pm \\
0.00^{\mathrm{a}}\end{array}$ & $\begin{array}{c}1047.34 \pm \\
74.00^{\mathrm{a}}\end{array}$ & $\begin{array}{c}100.00 \pm \\
0.00^{\mathrm{a}}\end{array}$ \\
\hline ZnONPs 250 & 100 & $\begin{array}{c}33.33 \pm \\
0.00^{\mathrm{ab}}\end{array}$ & $\begin{array}{c}81.02 \pm \\
5.61^{\mathrm{ab}}\end{array}$ & $\begin{array}{l}1.24 \pm \\
0.08^{\mathrm{bc}}\end{array}$ & $\begin{array}{l}3.22 \pm \\
0.10^{\mathrm{ab}}\end{array}$ & $\begin{array}{l}6.58 \pm \\
0.14^{\mathrm{ab}}\end{array}$ & $\begin{array}{c}96.34 \pm \\
2.11^{\mathrm{ab}}\end{array}$ & $\begin{array}{c}849.95 \pm \\
86.79^{\mathrm{b}}\end{array}$ & $\begin{array}{c}49.84 \pm \\
4.02^{\mathrm{b}}\end{array}$ \\
\hline ZnONPs 500 & 100 & $\begin{array}{c}30.56 \pm \\
4.81^{\mathrm{b}}\end{array}$ & $\begin{array}{c}63.99 \pm \\
5.84^{\text {cd }}\end{array}$ & $\begin{array}{c}1.57 \pm \\
0.14^{\mathrm{a}}\end{array}$ & $\begin{array}{c}2.86 \pm \\
0.13^{\mathrm{c}}\end{array}$ & & $\begin{array}{c}87.80 \pm \\
3.66^{\mathrm{c}}\end{array}$ & $\begin{array}{c}738.65 \pm \\
29.90^{\mathrm{bc}}\end{array}$ & $\begin{array}{l}42.24 \pm \\
5.31^{\text {bcd }}\end{array}$ \\
\hline ZnONPs 1000 & 100 & $\begin{array}{c}33.33 \pm \\
0.00^{\mathrm{ab}}\end{array}$ & $\begin{array}{c}79.55 \pm \\
13.78^{\mathrm{b}}\end{array}$ & $\begin{array}{c}1.29 \\
\pm 0.25^{\mathrm{bc}}\end{array}$ & $\begin{array}{c}3.17 \pm \\
0.29^{\mathrm{b}}\end{array}$ & $\begin{array}{l}6.50 \pm \\
0.43^{\mathrm{ab}}\end{array}$ & $\begin{array}{c}95.12 \pm \\
6.34^{\mathrm{ab}}\end{array}$ & $\begin{array}{c}686.11 \pm \\
61.38^{\mathrm{c}}\end{array}$ & $\begin{array}{l}40.51 \pm \\
2.57^{\text {cde }}\end{array}$ \\
\hline Cd 25 & 100 & $\begin{array}{c}30.56 \pm \\
4.81^{\mathrm{b}}\end{array}$ & $\begin{array}{c}75.19 \pm \\
4.49^{\mathrm{bc}}\end{array}$ & $\begin{array}{c}1.33 \\
\pm 0.08^{b}\end{array}$ & $\begin{array}{c}3.14 \pm \\
0.05^{\mathrm{b}}\end{array}$ & $\begin{array}{l}6.42 \pm \\
0.14^{\mathrm{b}}\end{array}$ & $\begin{array}{c}93.90 \pm \\
2.11^{\mathrm{b}}\end{array}$ & $\begin{array}{c}667.37 \pm \\
45.10^{\mathrm{c}}\end{array}$ & $\begin{array}{l}43.45 \pm \\
2.01^{\mathrm{bcd}}\end{array}$ \\
\hline Cd 50 & 100 & $\begin{array}{c}30.56 \pm \\
4.81^{\mathrm{b}}\end{array}$ & $\begin{array}{c}75.19 \pm \\
4.49^{\mathrm{bc}}\end{array}$ & $\begin{array}{l}1.33 \pm \\
0.08^{\mathrm{b}}\end{array}$ & $\begin{array}{l}3.14 \pm \\
0.05^{\mathrm{b}}\end{array}$ & $\begin{array}{l}6.42 \pm \\
0.14^{\mathrm{b}}\end{array}$ & $\begin{array}{c}93.90 \pm \\
2.11^{\mathrm{b}}\end{array}$ & $\begin{array}{c}655.24 \pm \\
72.36^{\mathrm{c}}\end{array}$ & $\begin{array}{c}36.44 \pm \\
6.34^{\mathrm{de}}\end{array}$ \\
\hline ZnONPs $250+$ Cd 25 & 100 & $\begin{array}{c}33.33 \pm \\
0.00^{\mathrm{ab}}\end{array}$ & $\begin{array}{c}75.19 \pm \\
4.49^{\mathrm{bc}}\end{array}$ & $\begin{array}{c}1.33 \pm \\
0.08^{\mathrm{b}}\end{array}$ & $\begin{array}{c}3.11 \pm \\
0.10^{\mathrm{b}}\end{array}$ & & $\begin{array}{c}93.90 \pm \\
2.11^{\mathrm{b}}\end{array}$ & $\begin{array}{c}742.94 \pm \\
68.44^{\mathrm{bc}}\end{array}$ & $\begin{array}{c}44.47 \pm \\
4.38^{\mathrm{bc}}\end{array}$ \\
\hline ZnONPs $250+$ Cd 50 & 100 & $\begin{array}{c}30.56 \pm \\
4.81^{\mathrm{b}}\end{array}$ & $\begin{array}{c}58.33 \pm \\
0.00^{\mathrm{d}}\end{array}$ & $\begin{array}{c}1.71 \pm \\
0.00^{\mathrm{a}}\end{array}$ & $\begin{array}{c}2.69 \pm \\
0.05^{\mathrm{c}}\end{array}$ & $\begin{array}{l}5.75 \pm \\
0.00^{\mathrm{c}}\end{array}$ & $\begin{array}{c}84.15 \pm \\
0.00^{\mathrm{c}}\end{array}$ & $\begin{array}{c}620.79 \pm \\
46.43^{\mathrm{c}}\end{array}$ & $\begin{array}{c}37.95 \pm \\
1.14^{\text {cde }}\end{array}$ \\
\hline ZnONPs $500+C d 25$ & 100 & $\begin{array}{c}33.33 \pm \\
0.00^{\mathrm{ab}}\end{array}$ & $\begin{array}{c}81.02^{\mathrm{a}} \\
5.61^{\mathrm{ab}}\end{array}$ & $\begin{array}{l}1.24 \pm \\
0.08^{\mathrm{bc}}\end{array}$ & $\begin{array}{l}3.22 \pm \\
0.10^{\mathrm{ab}}\end{array}$ & $\begin{array}{l}6.58 \pm \\
0.14^{\mathrm{ab}}\end{array}$ & $\begin{array}{c}96.34 \pm \\
2.11^{\mathrm{ab}}\end{array}$ & $\begin{array}{c}617.86 \pm \\
52.68^{\mathrm{c}}\end{array}$ & $\begin{array}{c}40.53 \pm \\
6.57^{\text {cde }}\end{array}$ \\
\hline ZnONPs $500+$ Cd 50 & 100 & $\begin{array}{c}30.56 \pm \\
4.81^{\mathrm{b}}\end{array}$ & $\begin{array}{c}61.87 \pm \\
3.06^{\mathrm{d}}\end{array}$ & $\begin{array}{c}1.62 \pm \\
0.08^{\mathrm{a}}\end{array}$ & $\begin{array}{c}2.81 \pm \\
0.05^{\mathrm{c}}\end{array}$ & $\begin{array}{c}5.92 \pm \\
0.14^{\mathrm{c}}\end{array}$ & $\begin{array}{c}86.59 \pm \\
2.11^{\mathrm{c}}\end{array}$ & $\begin{array}{c}714.44 \pm \\
66.44^{\mathrm{bc}}\end{array}$ & $\begin{array}{c}39.78 \pm \\
3.28^{\text {cde }}\end{array}$ \\
\hline ZnONPs $1000+$ Cd 25 & 100 & $\begin{array}{c}33.33 \pm \\
0.00^{\mathrm{b}}\end{array}$ & $\begin{array}{c}84.26 \pm \\
5.61^{\mathrm{ab}}\end{array}$ & $\begin{array}{l}1.19 \pm \\
0.08^{\mathrm{bc}}\end{array}$ & $\begin{array}{l}3.28 \pm \\
0.10^{\mathrm{ab}}\end{array}$ & $\begin{array}{l}6.67 \pm \\
0.14^{\mathrm{ab}}\end{array}$ & $\begin{array}{c}97.56 \\
\pm 2.11^{\mathrm{ab}}\end{array}$ & $\begin{array}{c}695.62 \pm \\
193.16^{\mathrm{c}}\end{array}$ & $\begin{array}{c}38.01 \pm \\
6.10^{\text {cde }}\end{array}$ \\
\hline ZnONPs $1000+$ Cd 50 & 100 & $\begin{array}{c}33.33 \pm \\
0.00^{\mathrm{ab}}\end{array}$ & $\begin{array}{l}81.67 \pm \\
10.10^{\mathrm{ab}}\end{array}$ & $\begin{array}{l}1.24 \pm \\
0.16^{\mathrm{bc}}\end{array}$ & $\begin{array}{l}3.22 \pm \\
0.19^{\mathrm{ab}}\end{array}$ & $\begin{array}{c}6.58 \\
\pm 0.29^{\mathrm{ab}}\end{array}$ & $\begin{array}{c}96.34 \pm \\
4.23^{\mathrm{ab}}\end{array}$ & $\begin{array}{c}673.24 \pm \\
48.52^{\mathrm{c}}\end{array}$ & $\begin{array}{c}34.95 \pm \\
4.60^{\mathrm{e}}\end{array}$ \\
\hline
\end{tabular}

Data are means \pm SD of three independent experiments.

$a, b, \ldots$ or ${ }^{g}$ indicated a significant difference at $\mathrm{p} \leq 0.05$.

Seed germination and seedling growth parameters are very important indicators in yield determination (Rauf et al., 2007). Dhanda et al. (2004) indicated that seed vigor index and shoot length are among the most sensitive indices to stress, followed by root length and coleoptiles length. In present study, the germination parameters presented in Table (3) indicated that all treatments led to $100 \%$ germination of seeds (FGP) and a significant reduction in all germination indices except MGT. Prapatsorn et al. (2011) found that ZnONPs didn't affect rice grains germination but, in a higher concentration reduced the root length. Seed germination needs water imbibitions to start a physiological process (Wierzbicka and Obidzinska, 1998). However, in case of germination seed of Faba bean take place normally but the bad effect is more obvious in the roots, probably due to the coat of faba bean seed, which can act to protect the embryo (Prapatsorn et al., 2011). Also, other nanoparticles like alumina (nano- $\mathrm{Al}_{2} \mathrm{O}_{3}$ ) at $2000 \mathrm{mgL}^{-1}$ could inhibit root elongation of five plant species, Zea mays (corn), Cucumis sativus (cucumber), Glycine max (soybean), Brassica oleracea (cabbage), and Daucus carota (carrot) (Yang and Watts, 2005). Uptake and movement of water in the embryo axis was inhabited by $\mathrm{Cd}$ concentration lowered the seedling development in Suaeda salsa seeds (Sujing et al., 2012). Many studied reported that $\mathrm{Zn}^{2+}$ showed an inhibitory effect on root growth and the cell division (Munzuroglu and Geckil, 2002; El-Ghamery et al., 2003; Talgar et al., 2011). This may be associated with several disorders in the event chain of germinative metabolism (Sujing et al., 2012). The inhibition of root elongation caused by heavy metal may be due to metal interference with cell division, in cluding inducement of chromosomal aberrations and abnormal mitosis (Talebi et al., 2014). Talgar et al. (2011) reported that, the reduction in the rate of mitotic division in garlic plant was attributed to the mitotic inhibition by ZnONPs, indicated that ZnONPs could interfere with the development of mitosis and cause cytotoxic effects. It might be due to 
the inhibition of DNA synthesis at S-phase or a blocking in the G2 phase of the cell cycle (Duan and Wang, 1995; Borboa and De la Torre, 1996; Sudhakar et al., 2001).

This evidence supporting that some engineered nanoparticles could exert physio-chemical toxicity or bad effect on plant depending on their chemical composition, size, surface energy and importantly is the species of plant which resulting in different ways (Prapatsorn et al., 2011).

\section{CONCLUSION}

It could be concluded that increase doses of ZnONPs decrease the content of $\mathrm{Cd}$ in the plant, which leads to reduce the Cd toxicity. Doses of ZnONPs (250, 500 and $1000 \mathrm{mg} \mathrm{L}^{-1}$ ) haven't positive effect on germination parameters. Effect of applications of nanotechnology in plant remained need more effort and more study to clear their benefits or harmful.

\section{REFERENCES}

Ahsan, N, DG Lee, SH Lee, KY Kang, JJ Lee, PJ Kim, HS Yoon, JS Kim and BH Lee (2007). Excess copper induced physiological and proteomic changes in germinating rice seeds. Chemosphere, 67(6): 1182-1193.

Auld, D. S. (2001). Zinc coordination sphere in biochemical zinc sites. Biometals, 14: 271-313.

Ball, P. (2002). Natural strategies for the molecular engineer. Nanotechnology, 13: 15 - 28.

Benavides, MP, SM Gallego and ML Tomaro (2005). Cadmium toxicity in plants. Braz J Plant Physiol., 17, $21-4$.

Borboa, L and C. De la Torre (1996). The genotoxicity of $\mathrm{Zn}$ (II) and $\mathrm{Cd}$ (II) in Allium cepa root meristematic cells. New Phytologis, 134: 481-486.

Dhanda, S.S., G.S. Sethi and R.K. Behl (2004). Indices of drought tolerance in wheat genotypes at early stages of plant growth. J. Agron. Crop. Sci., 190: 6-12.

Duan, CQ and HX Wang (1995). Cytogenetical toxical effects of heavy metals on Vicia faba and inquires into the Vicia-micronucleus. Acta Bot Sinica, 37: 14-24.

El-Ghamery, AA, MA El-Kholy and MA Abou ElYousser (2003). Evaluation of cytological effects of $\mathrm{Zn}^{2+}$ in relation to germination and root growth of Nigella sativa L. and Triticum aestivum L. Mutat Res., 537: 29-41.

Eshghi, S, MR Mahmoodabadi, GR Abdi and B Jamali (2010). Zeolite Ameliorates the Adverse Effect of Cadmium Contamination on Growth and Nodulation of Soybean Plant (Glycine max L.). J Biol Environ Sci., 4: 43-50.

Fargašová, A. (2004). Toxicity comparison of some possible toxic metals $(\mathrm{Cd}, \mathrm{Cu}, \mathrm{Pb}, \mathrm{Se}, \mathrm{Zn})$ on young seedlings of Sinapis alba L. Plant Soil Environ., 50(1): 33-38.

Gowayed, S. M. H. and O. A. Almaghrabi (2013). Effect of copper and cadmium on germination and anatomical structure of leaf and root seedling in Maize (Zea mays L). Australian Journal of Basic and Applied Sciences, 7(1): 548-555.
Hart, J. J., R. M. Welch, WA Norvell and L. V. Kochian (2002). Transport interactions between cadmium and zinc in roots of bread and durum wheat seedlings. Physiologia Plantarum, 116: 73-78.

Hasan, SA, Q. Fariduddin, B. Ali, S. Hayat and A. Ahmad (2009). Cadmium: Toxicity and tolerance in plants. J. Environ. Biol., 30(2): 165-174.

Hassan, M. J., G. Zhang, F. Wu, K. Wei and C. Chonghua (2005). Zinc alleviates growth inhibition and oxidative stress caused by cadmium toxicity in rice. Journal of Plant Nutrition and Soil Sci., 168: 256-261.

Hediat, M. H. Salama (2012). Effects of silver nanoparticles in some crop plants, Common bean (Phaseolus vulgaris L.) and corn (Zea mays L.). International Research Journal of Biotechnology, 3(10): 190-197.

Homa, M and R. Aghili (2014). Effect on Germination and Early Growth Characteristics in Wheat Plants (Triticum aestivum L.)Seeds Exposed to TiO Nanoparticles. Journal of Chemical Health Risks, 4(1): 29-36.

Jackson, M. L. (1973). Soil Chemical Analysis. Prentice-Hall of India Private Limited.

Jaouhra Cherif, Chamseddine Mediouni, Wided Ben Ammar and Fatma Jemal (2011). Interactions of zinc and cadmium toxicity in their effects on growth and in antioxidative systems in tomato plants (Solanum lycopersicum). Journal of Environmental Sciences, 23(5): 837-844.

Lin C.C. and C.H. Kao (1996). Disturbed ammonium assimilation is associated with growth inhibition of roots in rice seedlings caused by $\mathrm{NaCl}$. Plant Growth Regulation, 18(3): 233-238.

Lin, D. and B. Xing (2008). Root uptake and phytoxoxicityof $\mathrm{ZnO}$ nanoparticles.Environ. Sci. \& Tech., 42: 5580-5585.

Liu, TT, P Wu, LH Wang and Q Zhou (2011). Response of Soybean Seed Germination to Cadmium and Acid Rain. Bio Trace Elem Res., 144(1-3): 11861196.

Mahajan, Pramod, S. K. Dhoke, and A. S. Khanna (2011). Effect of Nano-ZnO Particle Suspension on Growth of Mung (Vigna radiata) and Gram (Cicer arietinum) Seedlings Using Plant Agar Method. Journal of Nanotechnology, Pp. 7, doi:10.1155/2011/696535

Munzuroglu, O. and H. Geckil (2002). Effects of metals on seed germination, root elongation, and coleoptile and hypocotyl growth in Triticum aestivum and Cucumis sativus. Arch Environ Contam Toxicol., 43: 203-213

Nel, A., T. Xia, L. Madler and N. Li (2006). Toxic potential of materials at the nanolevel. Science, 311: 622-627.

Pal, M, E. Horváth, T. Janda, E. Paldi and G. Szalai (2006). Physiological changes and defense mechanisms induced by cadmium stress in maize. J Plant Nutr Soil Sci., 169: 239-246.

Pramod, Mahajan, S. K. Dhoke and A. S. Khanna (2011). Effect of Nano-ZnO Particle Suspension on Growth of Mung (Vigna radiata) and Gram (Cicer arietinum) Seedlings Using Plant 
AgarMethod. Journal of Nanotechnology, Pp. 7, doi:10.1155/2011/696535.

Prapatsorn, Boonyanitipong, Prabhat Kumar, Boonthida Kositsup, Sunandan Baruah and Joydeep Dutta (2011). Effects of Zinc Oxide Nanoparticles on Roots of Rice Oryza Sativa L. International Conference on Environment and BioScience., IACSIT Press, Singapore, IPCBEE, 21: 172-176.

Prasad, T. N. V. K. V, P. Sudhakar, Y. Sreenivasulu, P. Latha, V. Munaswamy, K. Raja Reddy, T. S. Sreeprasad, P. R. Sajanlal and T. Pradeep (2012). Effect of nanoscale zinc oxide particles on the germination, growth and yield of peanut. Journal of Plant Nutrition, 35: 905-927.

Ramesh, M, K Palanisamy, K Babu and NK Sharma (2014). Effects of bulk \& nano-titanium dioxide and zinc oxide on physio-morphological changes in Triticum aestivum Linn. J Glob Biosci., 3: 415422.

Raskar, S and SL Laware (2013). Effect of titanium dioxide nano particles on seed germination and germination indices in onion. Plant Sciences Feed, 3(9): 103-107.

Raskar, SV and SL Laware (2014). Effect of zinc oxide nanoparticles on cytology and seed germination in onion. Int J Curr Microbiol App. Sci., 3: 467-473.

Rauf, M., M. Munir, M. UI-Hassan, M. Ahmed and M. Afzai (2007). Performance of wheat genotypes under osmotic stress at germination and early seedling growth stage. African J. of Biotechnology, 8: 971-975.

Roco, MC (2003). Broader societal issue on nanotechnology. Journal of Nanoparticle Research, 5: $181-189$.

Safaa, G. Kumari and Joop A.G. V Leur 2011. Viral diseases infecting faba bean (Vicia faba L.). Grain Legumes, 56: 24-26.

Sedghi, M., M. Hadi and SG Toluie (2013). Effect of nano zinc oxide on the germination of soybean seeds under drought stress. Ann West Uni Timis, oara ser Biol., XVI(2):73-78

Sfaxi-Bousbih, A., A. Chaoui and EIE Ferjani (2010). Cadmium impairs mineral and carbohydrate mobilization during the germination of bean seeds. Ecotox Environ Safe, 73(6): 1123-1129.
Siddiqui, Manzer H., Mohamed H. Al-Whaibi, Mohammad Firoz and Mutahhar Y. Al-Khaishany (2015). Nanotechnology and Plant Sciences Nanoparticles and Their Impact on Plants. Springer International Publishing Switzerland. Soil Science, 168: 256-261.

Sresty, T.V.S. and K.V.M. Rao (1999). Ultrastructural alteration in response to zinc and nickel stress in the root cells of pigeonpea. Environmental and Experimental Botany, 41: 3 -13.

Sudhakar, R, N. Gowda and G. Venu (2001). Mitotic abnormalities induced by silk dyeing industry effluents in the cells of Allium cepa. Cytologia 66: 235-239.

Sujing, Liua, Cuiyun Yang, Wenjun Xiea, Chuanhai Xiab and Ping Fanc (2012). The effects of cadmium on germination and seedling growth of Suaeda salsa. Procedia Environmental Sciences, 16: 293-298.

Talebi, S., S.M. N. Kalat and A.L.S. Darban (2014). The Study Effects of Heavy Metals on Germination Characteristics and Proline Content of Triticale (Triticoseale Wittmack). Intl J Farm \& Alli Sci., 3(10): 1080-1087.

Talgar, Shaymurat, Jianxiu Gu, Changshan Xu, Zhikun Yang, Qing Zhao, Yuxue Liu and Yichun Liua (2011). Phytotoxic and genotoxic effects of $\mathrm{ZnO}$ nanoparticles on garlic (Allium sativum L.): A morphological study. Nanotoxicology, 6(3): 241-8. doi:10.3109/17435390.2011.570462.

Waisberg, M, P. Joseph, B. Hale and D. Beyersmann (2003). Molecular and cellular mechanisms of cadmium carcinogenesis. Toxicology, 192: 95-17.

Wang, M. and Q. Zhou (2005). Single and joint toxicity of chlorimuronethyl, cadmium, and copper acting on wheat Triticum aestivum. Ecotoxicology and Environmental Safety, 60: 169-175.

Wierzbicka, M. and J. Obidzinska (1998). The effect of lead on seed imbibition and germination in different plant species. Plant Science, 137(2): 155171.

Yang, L. and Watts D. J. (2005). Particle surface characteristics may play an important role in phytotoxicity of alumina nanoparticles. Toxicology Letters, 158(2): 122-132. 